\title{
Civilisations
}

Revue internationale d'anthropologie et de sciences

humaines

63 | 2014

L'Ancestralité revisitée

\section{Les « os de la République » ou l'ancestralisation contestée au Congo-Brazzaville}

\section{Rémy Bazenguissa-Ganga}

\section{OpenEdition}

\section{Journals}

Édition électronique

URL : http://journals.openedition.org/civilisations/3645

DOI : 10.4000/civilisations.3645

ISSN : 2032-0442

\section{Éditeur}

Institut de sociologie de l'Université Libre de Bruxelles

\section{Édition imprimée}

Date de publication : 30 septembre 2014

Pagination : 27-44

ISSN : 0009-8140

Référence électronique

Rémy Bazenguissa-Ganga, «Les « os de la République » ou l'ancestralisation contestée au CongoBrazzaville », Civilisations [En ligne], 63 | 2014, mis en ligne le 30 septembre 2018, consulté le 30 avril 2019. URL : http://journals.openedition.org/civilisations/3645 ; DOI : 10.4000/civilisations.3645 


\title{
Les " os de la République" \\ ou l'ancestralisation contestée au Congo-Brazzaville*
}

\author{
Rémy BAZENGUISSA-GANGA
}

Résumé : Ce texte part d'une situation historique précise au Congo-Brazzaville inaugurée par deux événements simultanés de traitement symbolique des os des ancêtres. D'une part, l'édification d'un mausolée où ont été transférés les restes du fondateur du pays, le colonisateur Pierre Savorgnan de Brazza. De l'autre, de l'autorisation par les gouvernants, sans les honorer officiellement, de l'exhumation par les Congolais des restes de leurs parents, enfouis à même la terre lors des affrontements armés des décennies 1990-2000. L'étude de ces cas sert de prétexte pour interroger à travers une série de figures de disparus, l'ordre républicain du CongoBrazzaville de la période coloniale à nos jours.

Mots-clés : Congo-Brazzaville, ordre républicain, ancestralisation, morts, os.

Abstract: This text originates from a specific historical situation in Congo-Brazzaville inaugurated by two simultaneous instances of the symbolic treatment of bones of ancestors. Firstly, the construction of a mausoleum where the remains of the founder of the country, the colonizer Pierre Savorgnan de Brazza, were transferred. Secondly, the authorization by the gouverners, without formal recognition, for the exhumation, by the Congolese, of the remains of their parents, buried throught the decade-long armed confrontation of 1990-2000. These case studies serve as a pretext for questioning a series of missing figures, in the republican order of Congo-Brazzaville from the colonial period till the present-day.

Keywords: Congo-Brazzaville, Republican order, ancestralization, dead bodies, bones.

* Ce texte poursuit l'analyse parue sur le titre « The bones of body politic : thoughts on the Savorgnan de Brazza Mausoleum », International Journal of Urban and Regional Research, 2011, 35 (2), pp. 445-452. 
ans les années 2000, les Autorités du Congo-Brazzaville décident de célébrer, malgré de nombreuses controverses, le $126^{\mathrm{ème}}$ anniversaire de la fondation de la capitale à travers la figure du fondateur de l'ordre républicain colonial, Pierre Savorgnan de Brazza. Est exclu, lors des cérémonies, le roi sacré des Téké qui, pourtant, figure dans la scène primordiale de la colonisation celui qui, par un échange de sang et de terre, a rendu possible cette fondation.

Inscrire les pratiques d'ancestralisation dans cette contradiction fondamentale permet de saisir une partie de leurs spécificités dans les ordres coloniaux et postcoloniaux. C'est ainsi que nous en retrouvons les effets dans des configurations inédites comme, par exemple, celles des pratiques de traitement rituel des os des ancêtres où la mémoire coloniale entre en tension avec les phénomènes contemporains de guerre. En 2006, les gouvernants inaugurent un mémorial où ils transfèrent les restes de Pierre Savorgnan de Brazza et de tous les membres de sa famille. Or, à la même période, ces gouvernants autorisent, sans les honorer officiellement, l'exhumation des restes humains, enfouis à même la terre lors des affrontement armés des décennies 1990-2000, ayant conduit à l'instauration du régime politique actuel. Ces différences dans le traitement des os s'inscrivent dans le même présent historique quant à leur effectuation. Il convient de rappeler ici que ces pratiques d'ancestralisation avaient émergé bien avant. Certains faits renvoyant à la moyenne durée en sont des indices. En effet, durant l'époque coloniale, certains gouvernés devant les incertitudes portant sur sa mort, pourtant confirmée par les administrateurs coloniaux mais jamais prouvée, avaient déjà institué André Matswa comme le nom politique des os disparus en célébrant un culte préfigurant son retour comme Sauveur messianique tutélaire. Ainsi, une histoire singulière d'ancestralisation se dessine qui s'aligne sur des contraintes spécifiques et dessine un ordre particulier de succession.

La situation historique qui sert ici de point de départ et d'observation articule deux ordres du temps : la moyenne durée permet de comprendre la logique symbolique qui informe les occurrences des pratiques d'ancestralisation dans le présent historique. L'analyse comparative de trois cas de traitements des os - du Sauveur, du Fondateur et des morts anonymes - permet d'explorer, de manière dynamique, chaque pratique dans sa singularité mais aussi dans sa succession. En posant leur déroulement comme un principe important de compréhension me permet d'argumenter que leur occurrence est saisissable en référence à des phases précises. Je nomme phase ce qui permet de saisir les différentes intrigues historiques qui configurent ces pratiques d'ancestralisation. J'ai montré, dans des travaux antérieurs, l'intérêt de ce type d'analyse (BazenguissaGanga et al. 2013 : 57-92 ; Bazenguissa-Ganga 2009 : 133-152). Plus précisément, il est possible d'en délimiter deux ici, configurées alternativement par les paradigmes d'écriture et du sang (Bazenguissa-Ganga 2014 : 163-185).

Ce texte est composé de quatre parties. Dans la première, je spécifierai les effets de l'ancestralisation contestée dans la mise en place de l'ordre colonial républicain. Je reconstruirai ensuite et alternativement chaque phase. La deuxième partie, portant sur la phase configurée par le paradigme de l'écriture, montrera comment la tension autour de la revendication des os porte sur l'incertitude en ce qui concerne la mort du Sauveur. La seconde phase, configurée par le sang, est abordée en deux temps. Ainsi, la troisième partie reviendra sur l'officialisation du fondateur de l'ordre républicain et, 
enfin, la quatrième, éclairera le dédain officiel pour les os d'une grande partie des morts anonymes victimes des guerres de démocratisation.

\section{L'ancêtre colonial : le point de vue des colonisés et de leurs descendants}

Je m'intéresse ici non pas au corps mais aux os c'est-à-dire à ce qu'il en reste après décomposition. Les pratiques qui se rapportent à leur traitement ne sont pas spécifiques au Congo. En Afrique du Sud (Rasool 2008), par exemple, ils interviennent comme des éléments importants dans les pratiques de mémorisation et de réconciliation. Dans certains pays européens (Koselleck 1997 : 135-160 ; Mosse 1999 ; Jauffret 1997 ; Audouin-Rouzeau 2000 : 47-71), par exemple, les ossuaires et certains monuments aux morts sont des dispositifs officiels qui, à la fois, ressourcent l'identité nationale et conjurent le spectre de la Guerre mondiale. Des cas existent sur tous les continents qui confirment leur articulation au temps passé, présent ou futur. Une approche comparative s'avèrerait nécessaire pour distinguer parmi toutes celles qui ont, de manière évidente, un rapport à la question de l'ancestralisation. Tâche qu'il m'est difficile d'accomplir ici en raison du manque de données et du fait que cela dépasserait le cadre de mon propos. J'opterai, par contre, pour une étude de cas qui permettra de délimiter certains aspects de ces pratiques. À cet égard, le Congo-Brazzaville offre une perspective spécifique intéressante.

Mon propos porte sur le regard qui, à partir de la scrutation des os, institue la continuité d'un ordre républicain. Une question mérite d'être posée : qu'est-ce qui, à l'œil nu, ressemble plus à un os qu'un autre os. La réponse ouvre sur un type de connaissance bien plus symbolique que réelle. Ce regard ouvre des perspectives sur l'ossature symbolique du pays. L'énoncé les «o(r)s de la république » correspond, dans ce cas, à un jeu de mot permettant de mener une réflexion sur les différents types de traitement politique des os dans l'ordre républicain du Congo. Plus encore, ce regard permet de requalifier la vie et la mort à l'aune du politique.

Le Congo comme unité territoriale est créé en 1960 et vient prendre la place de ce qui, durant l'époque coloniale, était désigné comme étant le Moyen-Congo. Cette entité a d'abord été intégrée dans le Congo français, puis dans l'Afrique Équatoriale Française (AEF). Toutefois, utiliser le substantif Congo me permet de chercher les dispositifs de continuité au-delà des transformations « historiques », ici, à travers la lecture des pratiques de traitement des os. Plus précisément, le mémorial construit pour recueillir les restes de de Brazza ouvre une question centrale qui résonne avec la problématique de l'ancestralisation.

Pour éclairer cette assertion, il suffit de rappeler que S. de Brazza fonde en 1880, le Congo comme un ordre colonial républicain qui délimite l'espace dans lequel la question de l'ancestralité sera appréhendée ensuite. Je pars ici d'un constat qui a l'allure d'un truisme uniquement pour celui qui ne lit pas les enjeux cachés. Le Congo n'est pas un pays qui se réduit à la somme de "sociétés traditionnelles africaines » et/ ou de la France mais qui, au contraire, a émergé d'un coup sous la forme d'une totalité significative (Lévi-Strauss 1983 : XLVII-L). Pour saisir les modalités de construction de cette nouvelle création, il convient de dire qu'elles reposent sur des logiques de déterritorialisation et de reterritorialisation (Deleuze et Guattari 1972 : 163-324 ; Deleuze et Guattari 1980 : 434-527) où le colonisé n'est pas simplement un autochtone, 
mais qu'il porte déjà en lui une différenciation produite par le colonisateur. Ainsi, en dépit des critiques qu'elle a pu susciter, la célébration de la mémoire de S. de Brazza permet de rappeler qu'un ordre politique fondé par la colonisation intègre déjà en lui son extérieur. Et cela permet également de nuancer fortement, voire d'invalider les lectures en termes d'extraversion et/ou de syncrétisme. Les situations sont diverses et méritent d'être saisies de manière plus complète pour considérer comment, de manière très spécifique, dans la nouvelle totalité significative mise en place, l'autochtone disparaît politiquement et laisse vacante la place de l'ancestralisation pour que s'institue la continuité. Aussi, le cas congolais, malgré sa typicité, ouvre la réflexion sur des questions importantes à considérer, y compris dans des expériences coloniales où il n'y a pas de revendication pour les restes du « fondateur colon ».

L'utilisation d'une approche historique comparative fournit une aide précieuse pour éclairer ce point. Évoquons pour ce faire, la situation des Amériques, de l'Australie, ou encore de la Nouvelle-Zélande (Aschcroft, Griffiths et Tiffin 1989), où la prise de position des descendants des colonisateurs est toujours mise en avant. Nous savons que les élites de ces pays se considèrent également comme les rejetons d'une expérience coloniale, à la fois colonisateurs et décolonisateurs/décolonisés. Toutefois, ce processus de domination pousse tout le monde à entreprendre un travail crucial de production imaginaire d'un monde commun. Ce type d'opération s'est également déroulé en Afrique même s'il y est difficilement appréhendé. Ainsi, demeurés sur place, les Africains, en même temps qu'ils luttaient contre les colonisateurs extérieurs, se posaient, en réalité, eux-mêmes comme les héritiers d'un territoire autre que celui dans lequel leurs ascendants avaient vécu auparavant. Ce détour historique permet de justifier l'idée que le colonisé n'est jamais premier, il est déjà un (re-)produit. En sortant de la dynamique de l'autochtonie, de la terre, il se trouve projeté dans le temps de l'ancestralité, du pouvoir, des conditions symboliques d'incarnation de l'unité sociale et de sa perpétuation. Le dépassement de la dimension de la terre instaure un ordre où le colonisé figure une autre version du descendant du colonisateur lui-même. Le colonisateur le produit car tout se décline par rapport à l'espace-temps institué par la domination coloniale. Dans les processus de déterritorialisation-reterritorialisation qui mettent en place ce dispositif, le colonisé est donc le « rejeton » que le colonisateur ne reconnaît pas mais qui, non plus, ne se reconnaît pas comme rejeton du colonisateur. C'est, encore, pour une fois, cet espace-temps qu'il convient de circonscrire. Le saut sur place, pour conquérir le territoire, son identité, à la fois, contre l'envahisseur et contre son propre passé, est d'une violence extrême. C'est ce qui perdure et s'incarne dans l'ordre politique républicain.

$\mathrm{Au}$ Congo, S. de Brazza intervient comme une des figures centrales de la nouvelle totalité produite par la colonisation. Dans la mesure où il est le fondateur de cet ordre républicain national, il y est, à la fois, dedans et dehors. Il occupe, de fait, une position d'exception (Schmitt 1988 ; Schmitt 1999), de dehors dans le dedans - le dehors qui confirme et institue le dedans. Cet ordre républicain institué par S. de Brazza se perpétue. Il suffit, pour s'en rendre compte, d'indiquer qu'une déclinaison de son nom propre, Brazza-ville, désigne toujours la capitale du pays. Ce nom persiste, à la différence de ce qui se passe dans d'autres États qui débaptisèrent leur capitale ou leur pays pour signifier, symboliquement, la rupture avec l'ancien colonisateur. Pourtant, en dépit du maintien du nom colonial, le Congo-Brazzaville a été, de 1963 à 1977 en particulier, l'un 
des pays africains progressistes en pointe dans la lutte contre son ancien colonisateur, la France. Consacrer et installer officiellement les restes de de Brazza dans la capitale indique que la question coloniale continue à hanter et persiste comme un retour du refoulé, malgré les différentes dénégations et ruptures. Plus encore, cette action reverse la violence fondatrice, celle de la colonisation, dans la violence conservatrice à savoir celle du pouvoir congolais actuel (Benjamin 2000 : 210-243).

En réalité, cette cérémonie d'ancestralisation renvoie à une reconfiguration de la représentation de la scène primordiale. Dans celle-ci, toute l'expérience coloniale se trouve réduite à un échange entre le roi sacré ou Makoko des Téké, Ilo 1, et de Brazza. Aussi le rite d'ancestralisation de de Brazza, peut être cerné a contrario en revenant sur les revendications des opposants dénonçant l'oubli du Makoko dans cette procédure mémorielle. Ces derniers exigeaient que Ilo 1 soit célébré dans le même espace-temps et, qu'une statue soit érigée en son honneur à l'entrée du mémorial au côté de celle de de Brazza. Or, cette revendication n'a jamais été satisfaite.

Pour comprendre cet échec et plus encore son oubli par les gouvernants, il convient de revenir à une analyse des pratiques de la construction symbolique de la République au Congo. Celle-ci met également en scène le thème, classique en anthropologie, de la rencontre entre le monde du pouvoir venu de l'extérieur et le monde de la terre (Izard 1992). À cet égard, le traité signé entre Ilo 1, maître des lieux où a été érigée la capitale coloniale, et P. Savorgnan de Brazza est considéré comme l'acte de naissance du pays (Brunschwig 1972 ; Coquery-Vidrovitch 1972). Ce traité doublé d'un échange de sang entre eux et surtout d'un don d'une poignée de la terre du Makoko au colonisateur, fera des Téké les « possesseurs de la terre de tout le Congo », tandis que les Français figureront comme les « gens du pouvoir ». Or, pour les Congolais, le nom Téké renvoie à l'autochtonie. Il se réfère au pays et à l'identité de tout élément naturel - humain, animal, végétal - qui s'y trouve, considéré dans son « authenticité » en opposition aux espèces venues du dehors. Ce nom Téké, reconfiguré par l'expérience coloniale, s'avère ambigu car il est souvent perçu comme équivalent à l'état de sauvagerie. En ce sens, celui qui est Téké n'est pas légitimé pour gouverner le pays. Nous voyons poindre là des tensions entre un ordre régi par l'autochtonie et celui qui l'est par l'ancestralité. Ainsi, l'exclusion de Ilo 1 du rituel républicain d'ancestralisation semble redoubler ce qui s'est noué symboliquement lors de la rencontre entre ces univers.

Pour approfondir cette réflexion, j'ai déjà souligné que le Congo en tant qu'institution républicaine coloniale est le résultat d'une opération complexe et irréversible de déterritorialisation-reterritorialisation au terme duquel l'État s'extrait et se dégage d'un coup comme totalité significative. Je peux maintenant préciser que cette opération s'exprime aussi par la mise en évidence de deux processus fondamentaux, à la fois opposés et complémentaires : d'une part, la marginalisation des Téké, et, d'autre part, la disposition qui réserve aux Français et leurs avatars l'accès aux plus hautes fonctions d'autorité. Par ce double processus, le détenteur du pouvoir suprême se dote du moyen de l'exercer sans partage, en évacuant toute pression de la part des représentants de la terre.

L'évocation du mausolée et des os des anonymes renvoie à des pratiques d'ancestralisation lesquelles se réfèrent à la généalogie, fondent l'existence des chefs et introduisent directement dans certaines sphères de la personne. Ces pratiques sont 
configurées dans deux phases articulées, alternativement, par le paradigme de l'écriture et du sang que je vais maintenant aborder.

Tout d'abord, j'insisterai sur la difficulté de présenter toutes les personnes qui incarnent les deux paradigmes. Le texte est plus centré sur l'ancestralité et trop d'informations peuvent en rendre la lecture plus difficile. Je propose dans le tableau suivant une liste des figures présidentielles.

Figures présidentielles qui incarnent les différents paradigmes

\begin{tabular}{|c|c|c|c|}
\hline Paradigmes & Noms & Mandats & Formations \\
\hline \multirow{5}{*}{ Scripturaire } & Abbé F. Youlou & $\begin{array}{l}\text { Nov. 1958- } \\
\text { août } 1963\end{array}$ & Prêtre \\
\hline & A. Massamba-Débat & $\begin{array}{l}\text { Août } 1963- \\
31 \text { juillet } 1968\end{array}$ & Instituteur \\
\hline & $\begin{array}{l}\text { Capitaine } \\
\text { M. Ngouabi }\end{array}$ & $\begin{array}{l}31 \text { juillet } 1968- \\
\text { mars } 1977\end{array}$ & $\begin{array}{l}\text { Homme de troupe } \\
\text { dans caserne } \\
\text { puis formation } \\
\text { universitaire }\end{array}$ \\
\hline & $\begin{array}{l}\text { Général J. ombhi- } \\
\text { Opangault }\end{array}$ & $\begin{array}{l}\text { Mars 1977- } \\
\text { février } 1979\end{array}$ & $\begin{array}{l}\text { Homme de troupe } \\
\text { dans caserne }\end{array}$ \\
\hline & $\begin{array}{l}\text { Général D. Sassou- } \\
\text { Nguesso }\end{array}$ & $\begin{array}{l}9 \text { février 1979- } \\
1992\end{array}$ & $\begin{array}{l}\text { Instituteur puis } \\
\text { formation de } \\
\text { sous-officier }\end{array}$ \\
\hline \multirow{2}{*}{ Sang } & P. Lissouba & $\begin{array}{l}\text { 1992- } \\
\text { octobre } 1997\end{array}$ & Biologiste \\
\hline & $\begin{array}{l}\text { Général D. Sassou- } \\
\text { Nguesso }\end{array}$ & Depuis octobre 1997 & \\
\hline
\end{tabular}

La délimitation précise des chronologies évite à cette présentation d'être trop statique. D'une part, il apparaît que l'exercice de la fonction militaire n'est pas directement liée au paradigme du sang comme pour le commandant Ngouabi et les généraux Yombhi et Sassou. Jusque dans les années 1980, le paradigme scripturaire domine. De même, la formation universitaire ne l'est pas non plus pour le paradigme scripturaire. Le cas de P. Lissouba l'illustre bien. D'autre part, ce tableau souligne aussi le passage d'un paradigme à l'autre comme le marque, avec beaucoup de clarté, le cas de D. Sassou. Je ne m'appesantirai pas, par la suite, sur toutes ces figures mais plutôt sur celles autour desquelles le système symbolique de l'ancestralité se cristallise dans les deux paradigmes. 


\section{André Matswa ou le nom politique des os}

Pour expliciter la première phase configurée par l'ordre scripturaire, je reviens sur le cas qui a inauguré, pour les Congolais, la logique symbolique des différentes pratiques de traitements des os des ancêtres. En effet, la réclamation des restes du fondateur d'un ordre qui se perpétue en son absence, relève de pratiques anciennes dans l'histoire de moyenne-durée du Congo. Dans cet ordre d'idée, « Matswa » figure déjà, le nom politique des os.

Revenons au point de départ, à savoir la période qui survient après la Seconde Guerre mondiale. Une figure singulière, le «Kalaka », incarne, pour les Congolais, l'idéal de la citoyenneté ou des avatars du pouvoir qui vient du dehors. Kalaka vient de la traduction locale de deux substantifs français, « clerc » et « crack ». Sa signification oscille entre la compétence, la performance et l'élégance. Dans une très large mesure, les Kalaka constituent une noblesse d'État. Tous ont suivi une formation scolaire et/ ou professionnelle en internat public ou dans des casernes, pour les militaires, afin de constituer une élite subalterne et contribuer à la consolidation de la colonie. Soustraits à leurs familles, ils évoluent comme des orphelins, pris en charge par l'administration et se comportent, après leur réussite, avec arrogance. À certains égards, cette passion sociale exprime la manière de tenir leur place dans l'ordre colonial. Les colonisés non-Kalaka considèrent cette arrogance comme normale et attendent, en retour, que les Kalaka redistribuent à leur profit, grâce à l'application de leur savoir dans le gouvernement « éclairé » du pays, les avantages pour sortir de l'indigénat.

Les Congolais les surnomment « les écrivains »; ce qui manifeste une opération de transsubstantiation, dans la mesure où l'écriture y prend corps dans un groupe social. Le paradigme de l'écriture consolide l'expérience de la citoyenneté et, surtout, configure la crainte de la disparition du politique par celle du corps qui l'incarne. Ce paradigme excède, sous ses différentes formes, la matérialité de la feuille écrite et articule, de manière dynamique, deux ordres : l'impossible tentative des gouvernants de cacher les effets négatifs de leur domination sur le corps des gouvernés et les tactiques de ces derniers pour révéler les dessous de cette domination en y adhérant par la contestation. Différentes situations l'illustrent dans l'histoire politique congolaise. Tout d'abord, les Kalaka adressent aux administrateurs coloniaux, des lettres ouvertes de doléances contestant la situation déplorable des indigènes.

La Seconde Guerre mondiale ouvre aussi, dans le contexte français et le cadre du système colonial, une période de restructuration où les colonisés participent pleinement aux élections. Il est légitime de le qualifier d'ordre démocratique colonial républicain, d'autant plus que ce dispositif consacre, par sa périodicité, le vide du pouvoir (Lefort 1986). Cependant, au Congo, l'expérience de la vacuité du pouvoir s'exprime dans un différend entre les colonisateurs et les colonisés autour de la quête du corps d'un disparu, André Matswa (Balandier 1955 ; Sinda 1972). Ce dernier avait bénéficié d'une formation pour devenir catéchiste catholique. Il démissionne et s'engage comme employé aux écritures dans les douanes. Il se noue d'amitié avec les frères Tréchot, propriétaires de compagnies concessionnaires, qui le cooptent dans leur loge francmaçonnique. Il rejoint la métropole et s'engage dans l'armée, participe aux guerres coloniales (du Rif marocain, par exemple) et à la Première Guerre mondiale. Il obtient 
le grade de sergent. Son itinéraire montre que A. Matswa articule différents mondes sociaux : religieux, économique, guerrier, professionnel, etc.

A. Matswa, revendiquant une extension des droits politiques à tous les indigènes, se retrouve emprisonné à différentes reprises. Il meurt en captivité sans que son corps ne soit rendu aux siens pour être enseveli conformément aux rites consacrés. Ses geôliers l'enterrent en cachette. Cette situation crée un différend qui porte sur un doute. Alors que les colonisateurs le déclarent mort, les colonisés en exigent la preuve et ils construisent socialement leur contestation par la transfiguration d'un nom à travers l'évocation de ses os. Lors des consultations électorales périodiques, pendant quinze ans, près du tiers des électeurs écrivent, dans les isoloirs comme un acte magique, sur les bulletins, «bihissi $»$. Ce terme signifie, dans une des langues vernaculaires congolaises, les « os ». Cette inscription lapidaire ne renvoie pas seulement à une substancification mais à un questionnement : " puisque vous, les gouvernants, prétendez que Matswa est mort, alors montrez-nous ou rendez-nous ses os ?». La monstration des os doit prouver sa mort pour faire cesser la protestation; ce que les gouvernants ne font pas. Écrire le nom-question «bihissi » permet, en l'absence de réponse, d'engendrer le corps absent de Matswa. Par la suite, les Congolais transforment Matswa en Sauveur dans un culte messianique. Certains prétendent entretenir une relation épistolaire avec lui. De fait, le corps disparu du messie se transmue en corps écrit où le corps politique se reflète. L'écrivain ouvre ainsi, en déniant la question de la mort, l'expérience du double corps de celui qui ne meurt jamais et assure sa pérennité (Kantorowicz 1989).

En tant que Sauveur, Matswa/Bihissi est transporté, même si les Congolais l'imaginent en même temps captif, dans les sous-sols du Palais Bourbon, un autre monument massif. Le sous-sol indique que le lieu de prédilection de Matswa se trouve être les entrailles de la terre. On peut dire que le Palais Bourbon représente son tombeau. Dans la mesure où les Congolais dénient sa disparition physique, en l'absence de preuves, il vit toujours dans le politique. Matswa transcende la mort biologique et, pour cette raison, il exerce sa puissance sur l'ordre colonial. Selon les croyances, c'est lui qui, lors de la Seconde Guerre mondiale, aurait libéré la France grâce à son pouvoir mystique et, par la suite, facilité l'élaboration des lois qui aboutissent à l'Indépendance. Le regard qui insiste ici sur l'absence des os institue des phénomènes d'ancestralisation très intéressants. La suprématie de l'ordre visible dénie la disparition de Matswa et met en scène sa vie politique qu'il concentre dans le législatif. Là, celui qui bien qu'invisible et qui n'est qu'os revendiqué, inscrit la loi. Sous la pierre, il agit, cependant, dans la captivité.

Le questionnement qui porte sur les os éclaire donc la séparation entre la vie et la mort politique. Le type de reconnaissance qu'il implique ouvre sur un autre univers que je pose comme politique. Au-delà de la vie biologique, il est le produit de mobilisations sociales, de croyances, de réputations, d'espérance des gouvernés, etc. Évoluer dans cet univers, c'est, selon les cas, vivre ou mourir politiquement. Dans mes analyses antérieures (Bazenguissa-Ganga 1997), il est apparu que la plupart des expériences des Kalaka perpétuant l'histoire politique congolaise configurée par cette quête des os, suivait une même trajectoire. Le cheminement se décomposait en trois épisodes : la punition, le retrait et le retour. À un moment de sa carrière, activiste devenu opposant au régime, le kalaka subissait une répression (emprisonnement, limogeage, etc.) l'obligeant, par la suite, à se retirer de l'arène politique et, lorsque le régime qui 
l'avait persécuté s'affaiblissait, il revenait porté par des délégués l'ayant convaincu de remonter en selle. Le politique consiste donc, au Congo, au traitement spécifique de certains corps par la puissance publique qui les fait souffrir ou disparaître. Ces brutalités les dotent par effet induit, de puissance politique.

\section{Le traitement des os du fondateur de l'ordre colonial : le mausolée $S$. de Brazza}

La revendication des restes de de Brazza se cristallise dans une phase configurée par le sang. Je définirai, plus loin, de manière plus précise ce paradigme. La dynamique de construction du mausolée s'inscrit dans la logique du parcours de légitimité dessiné par l'ordre politique configuré par la quête des os du Sauveur. Il s'agit ici de traquer des logiques symboliques similaires. S. de Brazza, grand explorateur de la République française, ne bénéficie pas des fastes dignes de son activité. En total désaccord avec la manière dont le Congo français est exploité, il se retrouve en situation de disgrâce, d'où son retrait à Alger. Il meurt à Dakar, en retour de la mission d'évaluation des méfaits des compagnies concessionnaires dans le Congo français, et sa femme le fait enterrer à Alger. Cette dernière lance des accusations selon lesquelles son époux aurait été empoisonné par ses ennemis politiques et, plus particulièrement, par les propriétaires des compagnies concessionnaires. D'une manière ou d'une autre, de Brazza a vécu les deux étapes qui marquent une vie politique dans le sens congolais : la punition et le bannissement. De ce fait, que les gouvernants congolais réclament ses os, pour l'installer dans un espace public de pouvoir politique, correspond à une logique lui permettant d'accomplir la troisième étape du parcours de légitimité.

Posons le décor de manière plus précise (Martin-Granel 2010 : 293-307 ; Bernault 2010a : 367-390 et Bernault 2010b : 771-798 ; Tonda 2010 : 799-821). La construction du mémorial a été rendue possible, après des tractations entre les gouvernants congolais, avec le soutien de certains dignitaires français, les héritiers de de Brazza et les gouvernants algériens. Comme il s'avère ici plus instructif de saisir les dynamiques sociales en cause, il convient de s'appesantir sur le déroulement de ces tractations. Dans cette mesure, j'emprunterai quelques extraits parmi les informations agencées par un des héritiers de de Brazza (Serego Alighieri 2009).

Au cours de l'année 2000, la "Fondation Pierre Savorgnan de Brazza » est créée à Brazzaville pour mettre en valeur la mémoire de l'explorateur en vue du centenaire de sa mort. Le président en est le ministre d'État et chef de cabinet du président de la République, Emmanuel Yoka et Belinda Ayessa, la chargée de relations publiques. Sa création a été vraisemblablement inspirée par Jean-Paul Pigasse, le rédacteur en chef de l'agence de presse France-Adiac et conseiller du président congolais en tant que chargé de relations publiques. Au mois d'août 2003, Belinda Ayessa se rend au Frioul à la tête d'une délégation pour rendre visite au professeur Detalmo Pirzo, un des neveux de Savorgnan de Brazza, et l'invite à visiter le Congo sur les traces de son ancêtre. Il est, par la suite, coopté comme le représentant de la famille de Brazza au sein de la Fondation. Le professeur a mené plusieurs études en anthropologie sur les coutumes des populations d'Afrique subsaharienne. En septembre 2003, le professeur accepte de voyager au Congo et, pendant son séjour, il se rend à Mbé pour rendre visite au Makoko Gaston Ngouayoulou. Les deux hommes évoquent la mémoire de 
Pierre Savorgnan de Brazza et son désir d'être enterré en terre africaine. Ils s'accordent sur l'idée de transférer les dépouilles de l'explorateur d'Alger au Congo aux côtés de ceux de Ilo 1. Cette version est contredite dans les articles parus dans les Dépêches de Brazzaville, quotidien favorable au pouvoir, qui prétend que, dès le départ, c'est Detalmo qui désirait les transférer sur les rives du fleuve Congo. Les jours suivants, pendant une rencontre avec Denis SassouNguesso, Detalmo Pirzio-Biroli propose, d'ailleurs, au président congolais cette idée. La Fondation Pierre Savorgnan de Brazza est chargée d'organiser le transfert. En 2004, les autorités congolaises rendent publique leur intention de construire un mémorial à côté de la Mairie centrale de Brazzaville pour accueillir les restes de l'explorateur. Sa construction sera financée par l'État, la France et par des " généreuses contributions » d'un groupe de compagnies françaises, parmi lesquelles les groupes Total et Bolloré. Le 5 février 2005, Maurice Intsilambia, un Makoko usurpateur désigné par les dirigeants congolais, est invité par la Fondation Pierre Savorgnan de Brazza, pour participer à la cérémonie de pose de la première pierre du mausolée, en présence de DenisSassou-Nguesso, de Jacques Chirac et d'Omar Bongo. L'inauguration de la bâtisse est prévue le 14 septembre 2005 pour le centenaire de la disparition de de Brazza. Alors que la date prévue s'approche et que la construction du mausolée commence, l'initiative est de plus en plus critiquée par différents groupes : les Téké, outragés par l'exclusion du Makoko légitime; les opposants au régime congolais qui protestent contre le gaspillage des fonds publics; les intellectuels congolais (Obenga 2006 ; Atondi 2006) qui voient dans la célébration une tentative d'affirmer le "rôle positif de la colonisation »; les membres des Églises chrétiennes qui protestent contre leur exclusion des cérémonies orchestrées par le réseau des francs-maçons; enfin, les héritiers de de Brazza, autres que la branche représentée par Detalmo, qui jusque-là n'étaient pas au courant du transfert.

Du fait du retard des travaux, les gouvernants reportent l'inauguration du mausolée le 3 octobre pour le $126^{\text {ème }}$ anniversaire de la fondation de Brazzaville. Voici quelques extraits des cérémonies :

Le 2 octobre, la délégation arrive à l'aéroport de Brazzaville. Les autorités les accueillent. Les invités incluent huit représentants de la famille de Brazza, signataires de l'accord. Un groupe de représentants de la cour royale Téké, guidé par le premier vassal, est présent à l'accueil mais exclu des événements. La famille de de Brazza va rendre hommage aux représentants des Téké. Les cercueils de de Brazza, sa femme et de ses enfants, couverts d'un drapeau français, sont portés par un groupe de marins congolais, habillés comme des soldats coloniaux. Ils déposent les cercueils sur un véhicule. Une parade de voitures parcourt les boulevards de Brazzaville, remplis par une foule de curieux et par des groupes de danseurs en fête. Les cercueils sont amenés à l'Église du Saint-Esprit. Une messe y est célébrée par le nonce apostolique au Congo. Dans son prêche, ce dernier souligne que de Brazza a passé quelques années " en dehors de la grâce de Dieu », mais qu'il a su se repentir. Il évoque ainsi l'engagement de de Brazza dans la franc-maçonnerie auquel il a mis fin, en 1904, en raison des complicités de ses membres dans les brutalités des compagnies concessionnaires au Congo. Les cercueils sont ensuite déposés à la Mairie centrale, à côté du mausolée. Le 3 octobre, toutes les personnalités se retrouvent sur l'esplanade : Omar Bongo, Philippe Douste-Blazy, le ministre français des Affaires étrangères, JeanPaul Pigasse, Belinda Ayessa ; les représentants de la Fondation Brazza, les 
héritiers de de Brazza. Ces derniers occupent les sièges à côté de ceux destinés aux régnants Téké. Le Makoko Auguste Nguempio et la Ngalifourou Ngassa arrivent entourés par leur cour et par la musique et les danses des jours de fête. Ensuite, Denis Sassou-Nguesso fait son entrée, accompagné de sa femme et par la musique militaire. Avant de prendre place, sous les applaudissements du public, il rend hommage au Makoko et à la Ngalifourou. Après les allocutions de circonstances, les cercueils sont transportés dans le mausolée, suivis par SassouNguesso, Douste-Blazy et Bongo. Dans l'après-midi, les héritiers de de Brazza sont reçus dans le palais présidentiel. Un d'entre eux, Corrado Pirzio-Biroli donne, à l'étonnement de tous, au président un coffre avec les cendres de son père Detalmo, en lui priant de les déposer à l'intérieur du mausolée à côté des dépouilles de de Brazza. (En 2009, cette requête n'a pas encore été satisfaite; les cendres se trouvent auprès du nonce apostolique de Brazzaville). Les célébrations se concluent dans la soirée par un dîner organisé par la Présidence.

Toutes ces informations indiquent que les gouvernants congolais inaugurent le 3 octobre 2006, le mausolée. Or ce jour est celui de la fête de Brazzaville et non du centenaire de la disparition de l'ancêtre. La capitale se révèle ainsi comme l'élément principal dans ce dispositif de continuité qui implique les restes de l'ancêtre. D'ailleurs, les héritiers ont imposé une clause aux gouvernants qui spécifie que, d'aventure, si ses restes sont profanés qu'ils soient rapatriés à l'église Sainte-Anne. Cette clause semble indiquer que des incertitudes pèsent sur leur maintien dans ce lieu dans le cas où le pouvoir de Sassou-Nguesso prendra fin. Toutefois, il convient de souligner que cet édifice, l'un des plus imposants ayant été construit depuis la fin de la colonisation, occupe, avec la massivité de son marbre, le lieu où commença l'histoire nationale, l'endroit excentré où s'est noué le destin congolais. Ce monument prend la place laissée vide par la destruction des vestiges de la plus vieille maison coloniale du pays. Il surplombe le lac que forme le fleuve et tourne le dos à Kinshasa, la capitale-métropole du Congo-RDC. En dépit de son aspect massif, ce mausolée symbolise, en quelque sorte, le vide du lieu du pouvoir républicain.

Après ce rappel des faits, il convient maintenant d'entrer dans l'analyse des effets d'ancestralisation que recèle le mausolée. J'ai déjà posé que la revendication des restes de de Brazza se cristallise dans une phase configurée par le sang qui prend la place de celle qui l'était par l'expérience scripturaire. Il est maintenant temps de définir, plus précisément, le paradigme du sang. Ce dernier permet d'énoncer le lieu du pouvoir et ses mécanismes de reproduction en dehors de l'acquisition des atouts scolaires. Il établit, d'abord, avec évidence, que l'acte de verser du sang permet d'acquérir du pouvoir politique. En outre, de manière métaphorique, il configure aussi les pratiques de patrimonialisation et d'héritage. Il concerne, dans ce cas, l'expérience des individus qui reçoivent leur droit politique par le sang transmis par leur géniteur qui possède déjà ce droit. Cette donnée indique que le Congo passe d'une domination des Kalaka, dont la trajectoire sociale de réussite était marquée par une expérience d'orphelin, à celui des héritiers, des « enfants de... ». Le paradigme du sang, sous toutes ces réalisations, est, paradoxalement, lié au maintien de la vie au-delà de la mort " politique ». Contrairement au paradigme de l'écriture qui se transsubstantialisait en prenant forme dans un groupe social précis, celui du sang ne le fait pas. L'installation de la période de la démocratisation donne lieu à des compétitions pour des mandats électifs qui font, d'une part, émerger de nouveaux acteurs et, de l'autre, se poursuivre les affrontements 
armés - au sens réel et métaphorique - au-delà du moment électoral. Guerriers et francs-maçons dessinent deux types idéaux dans la mesure où les cas réels oscillent entre les deux. Le terme de guerrier émerge pour désigner toute personne impliquée, de manière réelle ou supputée, dans la série des conflits armés (Bazenguissa-Ganga et Yengo 1999 : 186-192 ; Bazenguissa-Ganga et al. 2013 : 57-92 ; Yengo 2006) lors des décennies 1990 et 2000 . Les guerriers y exercent un droit de vie et de mort. Les francs-maçons, par contre, rassemblent des individus qui, dans le cadre de la logique du partage des pouvoirs, contribuent au renforcement du présidentialisme. Appartenir à cette obédience indique les relations que chacun entretient avec le président de la République. Un tel engagement sous-entend surtout le désir de lui plaire.

Si l'on revient à la trajectoire de de Brazza, plusieurs similitudes se dessinent avec celle de A. Matswa. Tous deux appartiennent à l'armée et ont entretenu des relations avec les propriétaires des compagnies concessionnaires. Mais le trait le plus saillant se rapporte à leur appartenance religieuse, à la fois, au catholicisme et à la francmaçonnerie. La franc-maçonnerie contribue à intégrer, de manière plus large dans la phase articulée autour du paradigme du sang, cette ancestralisation à travers les stratégies mises en œuvre par les gouvernants pour embellir la capitale. En effet, le mausolée fait partie des constructions qui mettent en scène, dans la ville, les symboles maçonniques. Après les guerres électorales, les gouvernants réhabilitent, en quelque temps, grâce à la redistribution des recettes pétrolières, leurs anciennes demeures personnelles et/ou en construisent de nouvelles : espaces publics, ronds-points et bâtiments publics. Ces constructions se distinguent surtout par leurs signes maçonniques. Un triangle avec un œil au milieu surplombe leur devanture. Cet ornement permet à son propriétaire et/ou commanditaire de marquer son appartenance à l'élite politicoreligieuse. L'aménagement de l'espace public se conforme aussi à cette mode. Elle touche d'abord beaucoup de monuments officiels (Cour constitutionnelle, Palais de justice, etc.) et se dissémine dans tout le Congo dans le cadre des politiques publiques de « municipalisation accélérée ».

Les gouvernés voient fleurir ces bâtisses et la rumeur publique désapprouve. Les cas les plus significatifs visent la décision des autorités brazzavilloises de décorer, par des monuments, les ronds-points les plus prestigieux. Ces constructions obéissent principalement à deux logiques : la mise en scène historique de la ville et la figuration des pouvoirs de la franc-maçonnerie. La mise en scène consiste en l'érection de statues en mémoire de personnes disparues ayant occupé la fonction suprême de président du Conseil sous l'époque coloniale et de président de la République après l'indépendance du pays, mais également dans la présentation des armoiries de la République. Quant à la seconde logique, la rue congolaise retient l'utilisation des symboles égyptiens par les gouvernants. Tel est, par exemple, le cas de la statue $\mathrm{Ba}$, mi-oiseau et mi-homme, placée dans le centre ville, ou « La Ville ». Les gouvernés considèrent que cette statue entre dans une stratégie mise en œuvre par les gouvernants pour renforcer leur pouvoir sur la société. Un autre monument paraît également significatif, l'obélisque érigé au rond-point d'un quartier populaire, Moungali. Cet espace ne se trouve pas loin de l'Église Saint-Esprit où la dépouille de de Brazza a été célébrée. La logique de ces constructions publiques pour embellir la ville culmine dans le mausolée rempli des signes maçonniques.

Qu'en est-il du traitement des os des anonymes? 
Il est possible de retrouver la logique symbolique impulsée à partir de Matswa qui configure le traitement des os par les gouvernants, dans le Mausolée Savorgnan de Brazza en l'articulant avec la dispersion des os d'anonymes, non célébrés dans Brazzaville et dans une très grande partie du pays. Rappelons que l'inauguration du mausolée intervient après qu'une série de conflits armés ait divisé les populations congolaises. Ces affrontements interviennent dans le dispositif qui assure la sortie du système monopartiste et l'installation d'un ordre étatique régi par un régime multipartiste. Ce dispositif est aussi articulé sur des élections au suffrage universel.

Durant les conflits, une très grande partie de la population meurt victime de balles perdues ou des suites de maladie. Les rescapés évoquent, encore aujourd'hui, avec horreur et/ou stupeur, ou parfois même avec humour et/ou ironie, l'expérience éprouvante de l'enjambement des cadavres lors des fuites. Lorsque la situation le permettait, les vivants ensevelissaient les personnes tombées, que ces dernières appartiennent ou non, à leur famille. Dans la mesure du possible, ils marquaient les tombes de fortune par des signes. Pour ceux qu'ils connaissaient, ils s'empressaient d'avertir leurs proches du lieu de la sépulture. Chacun espérait ainsi qu'à la fin du conflit, tout le monde pourrait offrir une tombe digne à ses morts. Les corps, restés en terre trop longtemps, ont laissé place à un amas d'os après leur putréfaction. Combien de personnes ou d'os gisent dans ces différents endroits ? Personne ne peut le dire, mais Radio-trottoir ou la rumeur publique, quant à elle, révèle que certains lieux sont des « charniers » et d'autres, des tombes individuelles. Toutefois, rien ne prouve que les lieux identifiés contiennent des ossements, mais c'est ainsi que les habitants des quartiers, du moins, les appréhendent dans leur mémoire. J'ai recueilli de nombreux témoignages de découverte d'os. Les fortes pluies les font remonter à la surface et une grande partie de ces " charniers » deviennent des dépôts anarchiques d'ordure.

Lorsque les autorités étatiques autorisent enfin l'exhumation des corps disséminés dans le pays et leur enterrement dans les cimetières consacrés, s'ouvre une occasion pour les pratiques d'identification des lieux et de déplacement des restes mortuaires. Or, non seulement l'État ne prend pas en charge ces opérations, mais les familles doivent payer de fortes taxes pour les exhumations. Ces taxes servent en partie à rémunérer les services des Grandes Epidémies et de la Croix-Rouge congolaise qui interviennent pour désinfecter les lieux souillés par les morts. Parfois, des candidats aux législatives et municipales de 2005 se lancent, dans certains quartiers Sud de Brazzaville, dans ce travail nécrologique. Dans le cadre de leur campagne, ils prennent en charge quelques exhumations. Les différentes Églises chrétiennes s'y engagent également. Parallèlement, les familles s'évertuent à recueillir l'argent pour organiser les funérailles, les veillées, etc. Tous agissent ainsi au respect des obligations familiales.

La confrontation de ces morts anonymes, occasionnés par l'usage de la force publique par des civils congolais au nom de l'État, à la logique des parcours de légitimité, incite à poser les os des victimes comme étant investis d'une légitimité politique liée à la démocratisation. Et, c'est de cette légitimité dont il convient de rendre compte. Plus encore, puisque ces conflits armés installent un ordre étatique régi par un régime multipartiste articulé sur des élections au suffrage universel, il devient tout à fait pertinent d'interpréter ces affrontements armés comme le déroulement d'une étape du parcours de légitimité qui consacre la montée en puissance d'un nouveau sujet politique, le peuple « souverain ». Cette consécration s'opère de manière complexe. Les citoyens 
se mobilisent dans des groupes armés et exercent à l'encontre d'autres citoyens, leurs droits politiques nouvellement reconnus tout en le leur refusant. De plus, en exerçant, au nom de l'État, la force publique les uns contre les autres, ils se conforment au dispositif paradoxal de légitimation où les victimes sont aussi potentiellement investies de pouvoir politique que peut revendiquer une base qui, plus tard, se mobilisera autour de la cause que représentent leurs « souffrances ». Chose curieuse, aucune mobilisation n'a pris pour alibi les nombreux anonymes, tués isolément, pour construire cette expérience en cause politique. À telle enseigne qu'aucune manifestation, à l'égal de ce qui se passe dans les pays sud-américains par exemple, ne se déroule pour réclamer aux gouvernants, les corps des parents disparus. Ici, les pratiques de traitement des os aboutissent à la dépersonnalisation des disparus.

Étant donné que toute comptabilité macabre se révèle impossible, il reste, toutefois, l'alternative de cerner les attitudes des gouvernés, Brazzavillois par exemple, face à ces tombes de fortune renfermant des os investis de pouvoir politique. Je commencerai d'abord par considérer comment ils les traitent. J'ai déjà indiqué qu'une grande partie de ces «charniers » deviennent des dépôts anarchiques et illégaux d'ordure. Ce qui tendrait à signifier la souillure des lieux. Qu'en est-il, alors, des modalités de cohabitation entre les Brazzavillois et ces morts ? Il est assez frappant de voir qu'elle se déroule « pacifiquement ». Or, habituellement les morts font peur et hantent les vivants. Ces différentes interrogations que je posais à des interlocuteurs distincts ont suscité plusieurs réponses. La plus intrigante a consisté à souligner le fait que leur mort violente n'avait pas permis aux personnes malveillantes de prendre le contrôle de l'esprit du mort. Seule la mort advenue de manière lente permet de hanter les vivants. Dans ce cas, d'ailleurs, c'est l'enveloppe corporelle entière qui est utilisée. Cette carapace vide a l'aspect d'un zombie. À l'inverse, la mort violente sort le corps de cette logique sorcellaire. Certaines réponses soulignent, encore, la spécificité de ces morts : troués par les balles. D'autres évoquent la densité du nombre de morts qui rend, selon eux, insensible à cette catastrophe.

La non-inscription de ces disparus dans l'espace public évoque, clairement, l'expérience de la mort politique. Celle-ci est une mort que les os d'anonymes inscrivent dans la structure symbolique de la ville. Aucune stratégie de mémorisation ne leur est destinée. Ainsi, le mort politique est celui qui ne revient pas, ne hante pas les habitants, ou celui dont les os ne sont pas rituellement traités. Celui pour qui n'existe pas de mobilisation. Sa souffrance ne signifie rien, du point de vue collectif, pour les gouvernés. Plus encore, il n'a pas eu le temps de vivre la première et deuxième étape du parcours de légitimité : punition et retour en grâce. On peut dire qu'il meurt rapidement des suites de la punition exécutée par l'application de la force publique à son égard. Il git sans sépulture des suites de conflits électoraux en attente de l'effectuation possible de la troisième étape du parcours de légitimité.

La spécificité de la disparition des anonymes est différente de celles de Matswa et de Brazza. Même si la mort des anonymes est individuelle, elle intervient dans le cadre d'une série alors que Matswa et de Brazza sont des figures uniques lorsqu'ils disparaissent. Ils décèdent d'ailleurs durant la seconde étape de leur parcours de légitimité. Les anonymes quant à eux, disparaissent dès l'application de la punition et leur bannissement prend la forme de la mort biologique. En outre, les dépouilles des anonymes sont enterrées dans des tombes de fortune connues de certaines personnes, 
de manière individuelle et, souvent marquées par des ordures, etc. Leurs restes ne sont pas inscrits dans un ordre de signification scripturaire. Ils échappent donc aux modalités de la reconnaissance publique. Cependant, nul doute n'existe quant à la réalité de leur disparition. Du doute émerge, parfois, comme dans le cas de A. Matswa, l'attente messianique du retour.

\section{Retour sur la question de l'ancestralité}

Les os de la République contribuent, dans les pratiques d'ancestralisation contestée, à l'identification de valeurs qui reproduisent l'ordre mis en place sous la colonisation et imposent une dette à l'égard de la vie perdue à la suite de la violence d'État afin de conférer un sens à la survie de la nation. Aussi le questionnement qui porte sur eux éclaire ce qu'il convient d'entendre par la vie et la mort politiques. Au-delà du biologique, les mobilisations sociales, les croyances, les réputations, les espérances, etc., produisent ces dernières. Pour en saisir les modalités, j'insisterai sur l'importance de la succession comme mode d'explication. La consécration des restes dans le mausolée intervient dans le cadre du passage du paradigme de l'écriture à celui du sang. Ces paradigmes configurent deux expériences d'acquisition des atouts politiques : l'orphelinat vs l'inscription dans un ordre de parenté. En d'autres termes, le mérite ne compte plus mais la naissance. Que Savorgnan de Brazza ne soit pas transféré seul mais avec toute sa famille nucléaire en est un indice.

Le passage d'un paradigme à l'autre est aussi celui qui voit le relatif déclin des gouvernés dans la qualification des ancêtres à la montée en puissance des gouvernants. Lorsque les gouvernés influaient, une incertitude pesait sur la qualification politique de la mort, alors qu'avec les gouvernants, elle est attestée et ne souffre d'aucune contestation. Les gouvernants résolvent l'incertitude par des offres d'interprétation visuelle figurée par des monuments (mausolée et autres) dont la fonction est d'embellir les villes. Ils imposent ainsi un point de vue unique dans la perspective de l'ancestralité, celui de l'État. Dans la phase articulée sur l'écriture, la mort du Sauveur n'était pas encore revêtue de sa finitude immanente, cependant la disparition du sens transcendant de la mort ouvre la possibilité d'une prétention monopolistique dans l'autre phase.

Si on se concentre maintenant sur la phase articulée sur le sang, il convient de se demander, quels os instituent, dans le présent historique, par leur ancestralisation, le régime de la démocratisation. D'une part, la figure reconnue officiellement de de Brazza s'impose. Cependant, il convient de ne pas oublier les morts anonymes, même si ces derniers se retrouvent évincés de l'espace public. J'argumenterai en deux temps.

Tout d'abord, la différence de traitement indique que le point de vue des gouvernants évoque une séparation que des gouvernés contestent. En effet, les monuments funéraires célèbrent seulement les élites comme ancêtres, les gens de peu n'y existent guère. Or nous avons vu que dans cette phase, l'entreprise démocratique, en reconfigurant les logiques de parcours de légitimité, accélère la baisse de la transcendance et égalise le monde des disparus dans le sens où elle dissémine l'expérience de la mort surtout en touchant des anonymes. En effet, si nous considérons les ancêtres en tant qu'ils incarnent des vivants ou des morts, il faut prendre également en compte les anonymes et tenter de comprendre ce que leur ancestralisation, non officialisée, nous dit. Une comparaison entre les vivants et les morts politiques permet d'affirmer que l'expérience 
de la démocratisation exige des corps tuables qui ne nécessitent ni « sépulture », ni rituel national d'ancestralisation (Agamben 1998). Ces corps sacrifiés, victimes collatérales dont on ne réclame ni les corps ni un enterrement décent, n'entraînent aucune sanction. Leurs corps sortent de l'espace domestique et agissent, par leur invisibilité, dans l'espace public. Cette remarque aboutit à une contradiction : la démocratisation saisie à travers la logique des parcours de légitimité semble donc liée à l'expérience de la mort politique. Sans qu'il y ait là le moindre paradoxe, l'idéal démocratique a pour conséquence de permettre le sacrifice de vies humaines, même si l'on accorde une valeur à la vie du citoyen. Ne peut-on pas considérer ces morts comme des victimes sacrificielles qui instaurent un nouvel ordre social, leur sacrifice instituant la vie démocratique?

Ensuite, l'exclusion des anonymes se comprend aussi par une autre incertitude plus profonde qui conditionne celle sur la mort. Cette incertitude empêche la montée en puissance de la fonction du combattant, du soldat, mais concerne véritablement la dimension sacrificielle des anonymes. En effet, cette dimension apparaît avec évidence à partir de la comparaison de la situation des anonymes avec celle des « disparus du Beach ». Cette affaire a connu différents épisodes. Elle concerne la disparition de plus de trois cent jeunes hommes réfugiés au Congo-RDC. À la fin du conflit de 1998-2002, lorsqu'ils rentrent au Congo, avec l'approbation du gouvernement et du HCR, ils sont arrêtés au port de Brazzaville comme étant des miliciens de l'opposition infiltrés et ne sont plus reparus. Officiellement, nul ne connaît leur tombe, excepté Radio-trottoir qui charrie de nombreuses versions. Certaines prétendent qu'ils auraient été enterrés dans un conteneur et ensevelis dans la terre. Leurs parents se sont mobilisés et ont porté plainte. Cette affaire a été jugée en France et au Congo. Nous voyons poindre là une incertitude sur la qualité de combattant que l'affaire du Beach semble résoudre. En tant qu'ennemis armés, ces disparus sont « honorés » et présents dans l'espace public. Par contraste, la dimension sacrificielle s'éclaire car les anonymes ne relèvent pas de cette incertitude ce qui justifie leur exclusion. Ainsi lorsque, dans le cadre des processus d'ancestralisation, le point de vue des gouvernants semble résoudre l'incertitude sur la qualification politique de la mort, elle le fait en tournant le dos à une incertitude plus profonde qui renvoie à la qualification du combattant. Finalement dans les consécrations des ancêtres, articulées autour du mausolée, l'État en ne commémorant pas les victimes de mort violente, espère se mettre en représentation dans une généalogie pacifique. Cependant, la présence de ces morts anonymes réactive toujours la violence fondatrice qui évoque, tout en le surpassant, l'ordre colonial républicain.

Enfin, il reste à interroger le rapport différent à la « mort politique » entre, d'une part, le guerrier, soldat formé sur le front en dehors des casernes, et, de l'autre, les sacrifiés non revendiqués. Le guerrier est honni parce qu'il incarne la figure du proche (civil) en bourreau. Rappelons que la caserne, analogiquement à l'internat, renvoie à un ordre politique régi en dehors de l'ordre de la parenté. Ainsi, de manière paradoxale, le fait que le guerrier acquiert ses compétences hors de la caserne inscrit métaphoriquement son expérience dans l'ordre de la parenté. Elle est donc directement conforme au paradigme du sang. Par contre, même s'ils participent du même paradigme, les sacrifiés sortent de l'ordre de la parenté. En effet, de manière la plus significative, à la différence des « disparus du Beach », ils ne sont même pas revendiqués par leurs parents qui souvent ne savent pas où ils sont. Leur disparition évoque, de fait, une allégorie de l'internat et/ ou de la caserne. Ne doit-on pas considérer ainsi que les sacrifiés ré-installent, dans le 
sang, l'extériorité de l'écriture. Ils figurent ainsi, dans un registre amoindri, la victime idéale sous le modèle de A. Matswa à savoir de ces os perdus dans les entrailles de l'ancestralité. Le traitement différent de la mort politique entre les guerriers et les sacrifiés souligne que les deux paradigmes s'interpénètrent. À travers eux, l'ambiguïté et/ou la tension sang/écriture persiste dans l'ancestralité.

\section{Références citées}

Agamben, Giorgio, 1998. Homo sacer. Le pouvoir souverain et la vie nue. Paris : Seuil.

Aschcroft, Bill, Gareth Griffiths et Helen Tiffin, 1989. The Empire Writes Back: Theory and Practice in Post-Colonial Literatures. Londres et New York : Routledge.

Atondi, Lecas M., 2006. « Pouvoir congolais et révisionnisme postcolonial : le cas Pierre Savorgnan de Brazza ». Brazzaville : Multigraphié.

Audouin-Rouzeau, Stéphane, 2000. « Corps perdus, corps retrouvés. Trois expériences de deuils de guerre », Les Annales HSS, 1, pp. 47-71.

BALANDIER, Georges, 1955. Sociologie actuelle de l'Afrique noire. Paris : PUF.

Bazenguissa-Ganga, Rémy, 1997. Les voies du politique au Congo. Essai de sociologie historique. Paris : Karthala.

-, 2009. «Au-delà de l'Atlantique noir : les Afriques des banlieues « mondialisées », in C. Agudelo, C. Boidin et L. Sansone (coord.), Autour de l'Atlantique noir. Une polyphonie de perspectives, pp. 133-152. Paris : Éditions de l'IHEAL.

-, 2011. «The Bones of the Body Politic : Thoughts on the Savorgnan de Brazza Mausoleum », International Journal of Urban and Regional Research, 35 (2), pp. 445-452.

-, 2012. « Le meurtre du cardinal Émile Biayenda et la théologie politique du marxisme-lénisnisme au Congo », in R. Bazenguissa-Ganga, G. Sounga-Boukono et R. Tabard, Le cardinal Biayenda et le Congo-Brazzaville, pp. 51-97. Paris : Karthala.

-, 2014. « Beautifying Brazzaville : Arts of Citizenship in the Congo » in M. Diouf et R. Fredericks (éds), The Arts of Citizenship in African Cities. Infrastructures and Spaces of Belongings, pp. 163-185. New York : Palgrave MacMillan.

Bazenguissa-Ganga, Rémy et Patrice Yengo, 1999. « La popularisation de la violence au Congo », Politique africaine, 73, pp. 186-192.

Bazenguissa-Ganga, Rémy, et al., 2013. «Les « écuries » : les formes d'engagement des urbains dans la guerre électorale du Congo-Brazzaville (1997-2009) » in Rémy Bazenguissa-Ganga et Sami Makki (éds), Sociétés en guerres. Ethnographies des mobilisations violentes, pp. 57-92. Paris : Éditions de la MSH.

Benjamin, Walter, 2000. « Critique de la violence », Euvres 1, pp. 210-243. Paris : Folio-Gallimard.

Bernault, Florence, 2010a. «Colonial Bones : The 2006 Burial of Savorgnan de Brazza in the Congo », African Affairs, 109 (435), pp. 367-390.

-, 2010b. «Quelque chose de pourri dans le post-empire. Le fétiche, le corps et la marchandise dans le Mémorial de Brazza au Congo », Cahiers d'Études africaines, 198-199-200 (50 ans), pp. 771-798.

Brunschwig, Henri, 1972. Brazza explorateur. Les traités Makoko. Paris : Mouton et Cie.

Coquery-Vidrovitch, Catherine, 1972. Le Congo au temps des compagnies concessionnaires 1898-1930. Paris : Mouton et Cie.

Deleuze, Gilles et Félix Guattari, 1972. L'Anti Edipe, pp. 163-324. Paris : Minuit.

-, 1980. Mille Plateaux, pp. 434-527. Paris : Minuit, 1980. 
IzARD, Michel, 1992. L'odyssée du pouvoir. Un royaume africain : État, société, destin individuel. Paris : Éditions de l'EHESS.

JAUFFret, Jean-Charles, 1997. « La question du transfert des corps, 1915-1934 », in Rémy Cazals et Marc Ferro (éds), Traces de 14-18, actes du colloque international tenu à Carcassone du 24 au 27 avril 1996, pp. 133-146. Carcassone : Les Audois.

Kantorowicz, Ernst, 1989. Les deux corps du roi. Paris : Gallimard.

Koselleck, Reinhart, 1997. « Les monuments aux morts, lieux de fondation de l'identité des survivants », in Reinhart Koselleck, L'expérience de l'histoire, pp. 135-160. Paris : Gallimard-Le Seuil.

Lefort, Claude, 1986. Essais politiques. Paris : Points-Seuil.

LÉvi-Strauss, Claude, 1983. « Introduction à l'œuvre de Marcel Mauss », in Marcel Mauss, Sociologie et anthropologie, XLVII-L. Paris : PUF.

Martin-Granel, Nicolas, 2010. « Abracadabrazza » ou le roman du mémorial Pierre Savorgnan de Brazza », Cahiers d'Études africaines, 1 (197), pp. 293-307.

Mosse, George L., 1999. De la Grande guerre au totalitarisme. La brutalisation des sociétés européennes. Paris : Hachette.

OBEnGA, Théophile, 2006. « De Brazza, faux « humaniste », vrai « violeur »?»<www.congoplus.info>.

Rasool, Ciraj, 2008. « Bone Memory and the Disciplines of the Dead : Human Remains, Transitional Justice and Heritage Transformation in South Africa », in Martin Legassick et Ciraj Rassool, « South African Human Remains, Viennese Museums and the Politics of Repatriation : Reconsidering the Legacy of Rudolph Pöch », Unpublished paper presented to the symposium on The life of corpse, WISER, University of Witwatersrand, pp. 25-26.

Schмітт, Carl, 1988. Théologie politique. Paris : Gallimard.

-, 1999. La notion de politique. Théorie du partisan. Paris : Champs Flammarion.

Serego Alighieri, Pietro di, 2009. Chronique du transfert des dépouilles de Pierre Savorgnan de Brazza d'Alger à Brazzaville. Manuscrit inédit, $18 \mathrm{p}$.

TondA, Joseph, 2010. « Le Mausolée Brazza, corps mystique de l'État congolais ou corps du 'négatif' », Cahiers d'Études africaines, L (2-3-4), 198, pp. 799- 821.

Yengo, Patrice, 2006. La guerre civile au Congo-Brazzaville. Paris : Karthala. 